PROCEEDINGS OF THE

AMERICAN MATHEMATICAL SOCIETY

Volume 130, Number 2, Pages 367-370

S 0002-9939(01)06072-5

Article electronically published on June 8, 2001

\title{
SCHUR INDICES OF PERFECT GROUPS
}

\author{
ALEXANDRE TURULL
}

(Communicated by Stephen D. Smith)

\begin{abstract}
It has been noticed by many authors that the Schur indices of the irreducible characters of many quasi-simple finite groups are at most 2 . A conjecture has emerged that the Schur indices of all irreducible characters of all quasi-simple finite groups are at most 2 . We prove that this conjecture cannot be extended to the set of all finite perfect groups. Indeed, we prove that, given any positive integer $n$, there exist irreducible characters of finite perfect groups of chief length 2 which have Schur index $n$.
\end{abstract}

\section{INTRODUCTION}

The Schur index was introduced by Schur in 1906. If $\chi$ is an irreducible character of a finite group $G$, and $F$ is a field of characteristic zero, the Schur index $\mathrm{m}_{F}(\chi)$ of $\chi$ with respect to $F$ is the smallest positive multiplicity of $\chi$ in the character afforded by a $G$-module over the field $F$. We refer the reader to any standard character theory text, such as [2], for the basic properties of the Schur index.

Even though a well-known result of Brauer [1] shows that the Schur indices of irreducible characters of finite groups run through all the positive integers, a number of more recent results have given very small upper bounds for the Schur indices of the irreducible characters of many simple and quasi-simple groups. Thus, in [13], we give an algorithm to compute the Schur index of each irreducible character of any covering group of any finite alternating group, and obtain that it is 1 or 2 in each case. Similarly, in [14, we find an algorithm to calculate the Schur index of each irreducible character of the finite special linear groups, and they are all 1 or 2. More general results of Gow 6] and Ohmori [8, [10] show that, in general, the Schur index of any irreducible character of a finite quasi-simple classical group is always 1 or 2. These ideas, together with the results of Feit and Zuckerman [5], further imply that every irreducible character of a finite spin group has Schur index at most 2. These results show that the Schur indices for the ordinary covering groups of the classical groups are at most 2. For the exceptional groups of Lie type, Ohmori [9] proves that there exists an upper bound for the Schur indices of the irreducible characters in some cases. Furthermore, Feit [3], 4] has calculated the Schur indices for the covering groups of the sporadic simple groups, and again he obtains that the Schur indices are always at most 2 .

Received by the editors June 23, 2000 and, in revised form, July 14, 2000.

2000 Mathematics Subject Classification. Primary $20 \mathrm{C} 15$.

Key words and phrases. Brauer group, Schur index, linear groups, classical groups, characters, representations.

The author was partially supported by a grant from the NSA. 
These results (and others) have led some authors to conjecture that the Schur indices of every irreducible character of every covering group of every finite simple group will always be 1 or 2 . This conjecture remains open. A more general question has been proposed by P. Schmid [12], and repeated by U. Riese and P. Schmid [11]. Namely: Is the Schur index of every irreducible character of every finite perfect group always either 1 or 2? A perfect group is, of course, one which does not have any non-trivial abelian quotients.

In this paper, we settle in the negative the question of Schmid and Riese. We prove the following.

Theorem. Given any positive integer $n$, there exists some finite perfect group $G$ of chief length 2 and some irreducible character $\chi \in \operatorname{Irr}(G)$ such that $m_{\mathbf{Q}}(\chi)=n$.

\section{Proof of the Theorem}

Definition 1. Let $G$ be any finite group, let $N$ be a normal subgroup of $G$, and let $\psi \in \operatorname{Irr}(N)$. We define

$$
\mathcal{I}^{*}(\psi)=\left\{x \in G: \text { there exists } \sigma \in \operatorname{Gal}(\overline{\mathbf{Q}} / \mathbf{Q}) \text { such that } \psi^{x}=\sigma \psi\right\} .
$$

The content of the following lemma is included, for example, in [11, Theorem 1]. We include a short proof for the reader's convenience.

Lemma 2. Let $G$ be any finite group, let $N$ be a normal subgroup of $G$, and let $\psi \in \operatorname{Irr}(N)$. Then $\mathcal{I}^{*}(\psi)$ is a subgroup of $G$ containing $N$. Furthermore, if $\theta \in$ $\operatorname{Irr}\left(\mathcal{I}^{*}(\psi)\right)$ is such that $\operatorname{Res}_{N}^{\mathcal{I}^{*}(\psi)}(\theta)$ contains $\psi$, and $\chi=\operatorname{Ind}_{\mathcal{I}^{*}(\psi)}^{G}(\theta)$, then the following hold:

(1) $\chi \in \operatorname{Irr}(G)$.

(2) The field of definition of $\theta$ and that of $\chi$ coincide, that is $\mathbf{Q}(\theta)=\mathbf{Q}(\chi)$.

(3) Their Schur indices also coincide, that is $m_{\mathbf{Q}}(\chi)=m_{\mathbf{Q}}(\theta)$.

Proof. Since the action of $G$ on $\operatorname{Irr}(N)$ commutes with the action of $\operatorname{Gal}(\overline{\mathbf{Q}} / \mathbf{Q})$ on $\operatorname{Irr}(N)$, and this second action is itself commutative, it follows that $\mathcal{I}^{*}(\psi)$ is a subgroup of $G$ that contains the inertia group of $\psi$. Hence, by Clifford Theory, induction provides a one-to-one correspondence between the irreducible characters in $\operatorname{Irr}\left(\mathcal{I}^{*}(\psi)\right)$ whose restriction to $N$ contains $\psi$ and the irreducible characters in $\operatorname{Irr}(G)$ whose restriction to $N$ contains $\psi$. It follows immediately that $\chi \in \operatorname{Irr}(G)$ and that $\mathbf{Q}(\chi) \subseteq \mathbf{Q}(\theta)$. Let $\sigma \in \operatorname{Gal}(\mathbf{Q}(\theta) / \mathbf{Q}(\chi))$. Any irreducible character in the restriction of $\theta$ to $N$ is $G$-conjugate to $\psi$, so any irreducible character of the restriction of $\chi$ to $N$ is $G$-conjugate to $\psi$. It follows, since $\sigma \chi=\chi$, that $\sigma \psi=\psi^{x}$ for some $x \in G$. Hence, $x \in \mathcal{I}^{*}(\psi)$. Therefore, $\sigma \theta \in \operatorname{Irr}\left(\mathcal{I}^{*}(\psi)\right)$ is an irreducible character which induces to $\chi$ and whose restriction to $N$ contains $\psi$. By the uniqueness in Clifford Theory, it follows that $\sigma \theta=\theta$. Hence, $\sigma=1$ and $\mathbf{Q}(\theta)=\mathbf{Q}(\chi)$. Set $K=\mathbf{Q}(\chi)=\mathbf{Q}(\theta)$ and set $m_{1}$ and $m_{2}$ to be the Schur indices of $\theta$ and of $\chi$ respectively. We know there will be modules $M_{1}$ and $M_{2}$ over $K$ affording the character $m_{1} \theta$ and $m_{2} \chi$ respectively. Induction shows that there is a module over $K$ affording the character $m_{1} \chi$, and it follows that $m_{1} \geq m_{2}$. Restriction shows that there is a module over $K$ for $\mathcal{I}^{*}(\psi)$ whose character is such that the multiplicity of $\theta$ in it is $m_{2}$. Hence, by definition of Schur index, $m_{1} \leq m_{2}$. Hence, $m_{1}=m_{2}$. This concludes the proof of the lemma.

Lemma 3. Let $n$ be a positive integer and let $q$ be a finite prime such that $n$ divides $q-1$ but $(n,(q-1) / n)=1$. Suppose we have the following: 
(1) $G$ has a normal subgroup $N$ such that $|N|=q, G / N$ is cyclic of order $n^{2}$, and $\left|G / \mathbf{C}_{G}(N)\right|=n$.

(2) $C=\mathbf{C}_{G}(N)$ is cyclic of order $n q$.

(3) $\psi \in \operatorname{Irr}(C)$ is faithful, and we set $\theta=\operatorname{Ind}_{C}^{G}(\psi)$.

Then $\theta \in \operatorname{Irr}(G)$ and $m_{\mathbf{Q}}(\theta)=n$.

Proof. The inertia group of $\psi$ is $C$, so that $\theta \in \operatorname{Irr}(G)$. The Schur index of $\theta$ can be obtained, for example, from [1].

We are now ready to prove our theorem. We state it slightly more precisely than above.

Theorem 4. Given any positive integer $n$, there exists some finite perfect group $G$ of chief length 2 and some irreducible character $\chi \in \operatorname{Irr}(G)$ such that $m_{\mathbf{Q}}(\chi)=n$. Furthermore, if $N$ is the socle of $G$, we may take $N$ to be elementary abelian and $G / N$ to be isomorphic to $\mathbf{P S L}(2, p)$ for some odd prime $p$.

Proof. Since it is easy to find examples of characters of $\mathbf{S L}(2, p)$ of Schur index 1 and Schur index 2, we assume $n>2$. By Dirichlet's Theorem, we may take a prime $p$ such that $2 n^{2} \mid p+1$. Set $S=\mathbf{P S L}(2, p)$. Again, by Dirichlet's Theorem, let $q>|S|$ be a prime such that $n \mid q-1$ but $(n,(q-1) / n)=1$. This can be obtained, for example, by taking $q$ to be congruent to $n+1$ modulo $n^{2}$.

Let $N$ be the Steinberg module for $S$ over $\mathbf{F}_{q}$. It is the submodule of the natural permutation module of $S$ over $\mathbf{F}_{q}$ on the subspaces of $\mathbf{F}_{p}^{2}$ of dimension 1 of all vectors whose sum of coefficients is 0 . It is an irreducible module of dimension $p$ over $\mathbf{F}_{q}$. Let $C=\langle c\rangle$ be a Singer cycle of $S$. Then $|C|=(p+1) / 2$, and $C$ permutes freely the 1-dimensional subspaces of $\mathbf{F}_{p}^{2}$. Hence, every irreducible character of $C$ is afforded by some submodule of $\operatorname{Res}_{C}^{S}(N) \otimes \overline{\mathbf{F}}_{q}$. In particular, there exist some $v \in N$ such that $c v=\lambda v$, where $\lambda \in \mathbf{F}_{q}^{\times}$has order $n$. Furthermore, by Maschke's Theorem, there exists some $C$-submodule $N_{0}$ of $N$ such that $N=\langle v\rangle \oplus N_{0}$.

Set $G=S N$ to be the semidirect product. Then $G$ is clearly a finite perfect group of chief length 2. Let $\psi: N \rightarrow \mathbf{C}^{\times}$be defined by $\psi\left(\alpha v+n_{0}\right)=\zeta_{q}^{\alpha}$ for all $\alpha \in \mathbf{F}_{q}$ and all $n_{0} \in N_{0}$, where $\zeta_{q}$ is a primitive $q$-th root of 1 . Then $\psi$ is a linear character of $N$.

Let $I^{*}=\mathcal{I}^{*}(\psi)$. By our choice, $C N \subseteq I^{*}$. Suppose that $C N \neq I^{*}$. Then, by Dickson's Theorem (see for example (8.27) in [7]), since $n^{2} \geq 9, I^{*} \cap S$ contains a dihedral subgroup $D$ containing $C$ as a subgroup of index 2 . Since the kernel of $\psi$ is $N_{0}$, we have that $I^{*} \subseteq N_{G}\left(N_{0}\right)$. Hence, $I^{*}$ acts on $N / N_{0}$, a cyclic group of order $q$. It follows that the derived subgroup $D^{\prime}$ acts trivially on $N / N_{0}$. But $D^{\prime}$ is a subgroup of index at most 2 of $C$, and, therefore, as $n \geq 3$, it follows that $D^{\prime}$ is acting non-trivially on $N / N_{0}$, a contradiction. Hence, $I^{*}=C N$.

It now follows that the inertia group $I$ of $\psi$ is $I=\left\langle c^{n}\right\rangle N$. Let $\phi: I \rightarrow \mathbf{C}^{\times}$be defined by $\phi\left(c^{n \alpha}, \beta v+n_{0}\right)=\zeta_{n}^{\alpha} \zeta_{q}^{\beta}$, for all $\alpha, \beta \in \mathbf{Z}$ and $n_{0} \in N_{0}$, where $\zeta_{n}$ is a primitive $n$-th root of 1 . Hence $\phi \in \operatorname{Irr}(I)$ is an irreducible character of the inertia group of $\psi$ lying over $\psi$. Let $\theta=\operatorname{Ind}_{I}^{I^{*}}(\phi)$. Now $I^{*} / \operatorname{ker}(\theta)$ is a group satisfying the hypothesis of Lemma 3. Hence $\theta \in \operatorname{Irr}\left(I^{*}\right)$ and $\mathrm{m}_{\mathbf{Q}}(\theta)=n$. Set $\chi=\operatorname{Ind}_{I^{*}}^{G}(\theta)$. Then, by Lemma 2, $\chi \in \operatorname{Irr}(G)$ and $\mathrm{m}_{\mathbf{Q}}(\chi)=n$. This concludes the proof of the theorem.

Remark 5. The character $\chi$ we constructed has degree $n p(p-1)$, and its field of definition is the fixed field in $\mathbf{Q}\left(\zeta_{n}, \zeta_{q}\right)$ of the Galois action that fixes $\zeta_{n}$ and sends 
$\zeta_{q}$ to $\zeta_{q}^{\lambda}$. It is a subfield of index $n$ in $\mathbf{Q}\left(\zeta_{n}, \zeta_{q}\right)$. The choice of the Steinberg module for $N$ is more a convenience, but we had to take some care in choosing the prime $p$ and, especially, the prime $q$.

\section{REFERENCES}

[1] R. Brauer, Untersuchungen über die arithmetischen Eigenschaften von Gruppen Substitutionen II, Math. Z. 31 (1930), 733-747.

[2] W. Feit, Characters of finite groups, Benjamin, New York, Amsterdam, 1967. MR 36:2715

[3] W. Feit, The computations of some Schur indices, Israel J. Math. 46 (1983), 274-300. MR 85j:20004

[4] W. Feit, Schur indices of characters of groups related to finite sporadic simple groups, Israel J. Math. 93 (1996), 229-251. MR 96m:20022

[5] W. Feit and G. J. Zuckerman, Reality properties of conjugacy classes in spin groups and simplectic groups, Algebraists' homage: Papers in Ring Theory and Related Topics, Contemp. Math., vol. 13, Amer. Math. Soc., Providence, Rhode Island, 1982. MR 84f:20052

[6] R. Gow, On the Schur indices of characters of finite classical groups, J. London Math. Soc.(2) 24 (1981), 135-147. MR 82i:20012

[7] B. Huppert, Endliche Gruppen I, Springer-Verlag, Berlin, Heidelberg, New York, Tokyo, 1967. MR 37:302

[8] Z. Ohmori, On the Schur indices of certain irreducible characters of simple algebraic groups over finite fields, Proc. Japan Acad. Series A 64 (1988), 253-255. MR 89j:20045

[9] Z. Ohmori, On the upper bounds of the Schur indices of simple finite groups of Lie type, Proc. Japan Acad. Series A 72 (1996), 160-161. CMP 97:04

[10] Z. Ohmori, On the Schur indices of certain irreducible characters of finite Chevalley groups, Hokkaido Math. J. 28 (1999), 39-55. MR 2000d:20009

[11] U. Riese and P. Schmid, Schur indices and Schur groups, II, J. Algebra 182 (1996), 183-200. MR 97e:20009

[12] P. Schmid, Schur indices and Schur groups, J. Algebra 169 (1994), 226-247. MR 95i:20012

[13] A. Turull, The Schur index of projective characters of symmetric and alternating groups, Annals of Math. 135 (1992), 91-124. MR 93c:20026

[14] A. Turull, The Schur indices of the irreducible characters of the special linear groups, J. Algebra 235 (2001), 275-314.

Department of Mathematics, University of Florida, Gainesville, Florida 32611

E-mail address: turull@math.ufl.edu 